\title{
Demonstration of a sub-picosecond x-ray streak camera
}

Z. Chang, ${ }^{\text {a) }}$ A. Rundquist, J. Zhou, ${ }^{\text {b) }}$ M. M. Murnane, ${ }^{\text {c) }}$ and H. C. Kapteyn

Center for Ultrafast Optical Science, The University of Michigan, Ann Arbor, Michigan 48109-2099

\section{Liu, B. Shan, J. Liu, L. Niu, M. Gong, and X. Zhang \\ State Key Laboratory of Transient Optics Technology, Xi'an Institute of Optics and Precision Mechanics, Xi'an 710000, People's Republic of China}

(Received 11 March 1996; accepted for publication 6 May 1996)

\begin{abstract}
A novel design, magnetically focused, $x$-ray streak camera was designed and tested using sub-20 fs soft-x-ray pulses generated by high harmonic emission in a gas. The temporal resolution of the camera was demonstrated to be under $0.9 \mathrm{ps}$ throughout the ultraviolet to soft-x-ray wavelength region. Our streak camera represents the fastest x-ray detector developed to date. (C) 1996 American Institute of Physics. [S0003-6951(96)03327-X]
\end{abstract}

During the past decade, the development of ultrafast $\mathrm{x}$-ray sources based on laser-produced plasmas, high harmonic emission, and synchrotrons, has advanced rapidly. ${ }^{1-3}$ It is now possible to generate sub-picosecond pulses throughout the vacuum ultraviolet and x-ray region of the spectrum, and sub-10 fs pulses have been generated using high harmonic emission. ${ }^{4}$ However, progress in the development of ultrafast sub-picosecond x-ray detectors has been relatively slower. ${ }^{5,6}$ Although cross-correlation techniques have demonstrated femtosecond time resolution, such measurements are very difficult, and are possible only at discrete wavelengths. ${ }^{7}$ The fastest $\mathrm{x}$-ray streak camera measurement to date was demonstrated to be 2 ps. This measurement was limited both by the time response of the streak camera itself, and by the laser-plasma-based $\mathrm{x}$-ray source. ${ }^{5,6}$ In this letter we describe the design and implementation of a novel x-ray streak camera, which exhibits sub-picosecond time resolution. The time response of the streak camera was measured using ultrashort sub-20 fs high-order harmonics produced by a 25 fs laser. ${ }^{4,8}$ The resolution of our camera was experimentally demonstrated to be 0.88 ps.

It is well known ${ }^{9,10}$ that the temporal resolution of streak cameras is limited mainly by the transit time dispersion of the photoelectrons as they travel from the photocathode to the deflection plates. It is also limited by the spatial resolution, and the deflection speed of the streak plates. For subpicosecond time resolution, space-charge effects may also limit the time resolution, and thus limit the dynamic range. For our work, we designed and tested a novel streak camera design to reduce the limitations on temporal resolution as much as possible. The configuration of the new x-ray streak camera is shown schematically in Fig. 1. In our camera, a pair of meander-type deflection plates is located before a magnetic focusing lens. This has several advantages: first the electron transit time from the anode to the deflection plates is minimized, as is the transit-time dispersion; second, the fast time response and high deflection sensitivity $(8 \mathrm{~cm} / \mathrm{kV})$ of the meander-type deflection plates provides the possibility of high sweep speeds on the exit phosphor screen; finally, the

\footnotetext{
a) On leave from Xi'an Institute of Optics and Precision Mechanics, P. R. China.

${ }^{b}$ Present address: Dept. of Chemistry, M. I. T., Boston, MA 02139.

${ }^{c}$ Electronic mail: murname@eecs.umich.edu
}

short transit time also reduces space-charge effects.

The electron transit time dispersion from the photocathode to the deflection plates in the camera can be evaluated analytically. In the photocathode to anode region, it is straightforward to show that the transit time difference between an electron liberated with energy $e V_{0}$, and an electron liberated with zero energy is given by ${ }^{10}$

$$
t_{\mathrm{pa}}^{\prime}=\sqrt{\frac{2 m V_{0}}{e}} \frac{1}{E}
$$

where $m$ and $e$ are the charge and mass of the electron, respectively, $e V_{0}$ is the initial energy of an emitted photoelectron in the axial direction, and $E$ is the extraction field.

For x-ray photocathodes in the $100 \mathrm{eV}$ to $10 \mathrm{KeV}$ region, the distribution of the initial energies of the emitted photoelectrons can be expressed as ${ }^{11}$

$$
N\left(e V_{0}\right) \propto \frac{e V_{0}}{\left(e V_{0}+W\right)^{4}},
$$

where $W$ is related to the photocathode material $(W=1 \mathrm{eV}$ for $\mathrm{KBr}$ ). The photocathode material also determines the full width at half-maximum (FWHM) of the energy distribution $(\delta \epsilon)$. From Eqs. (1) and (2), we obtain the transit time distribution of the electrons. The FWHM of this distribution is defined as the time dispersion, and can be shown to be

$$
\delta t_{\mathrm{pa}}=\frac{2.63 \sqrt{\delta \epsilon}}{E}(p s)
$$

where $\delta \epsilon$ is in $\mathrm{eV}$, and $E$ is in $\mathrm{kV} / \mathrm{mm}$. For our camera, $\delta \epsilon=1.1 \mathrm{eV}$ (KBr photocathode), and $E=10 \mathrm{kV} / \mathrm{mm}$, resulting in a calculated time dispersion of about $276 \mathrm{fs}$.

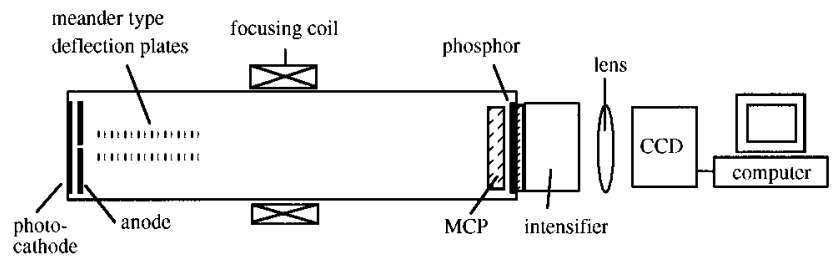

FIG. 1. Configuration of the sub-picosecond x-ray streak camera. 
In the nearly field free region between the anode and the entry to the deflection plates, we can again calculate the transit time distribution of the electrons. The distribution FWHM is then given by

$$
\delta t_{\mathrm{ad}}=\frac{1}{2} t_{\mathrm{ad}} \frac{\delta \epsilon}{e V_{a}},
$$

where $t_{\text {ad }}$ is the transit time for electrons with $\mathrm{eV}_{0}=0$. For our streak camera, $t_{\mathrm{ad}}=500 \mathrm{ps}$, so that $\delta t_{\mathrm{ad}}$ is $25 \mathrm{fs}$. It is clear that the time dispersion in the anode-to-deflection plates region is much smaller than in the photocathode-to-anode region. The above analysis does not take into account the angular distribution of the photoelectrons emitted from the photocathode. We have, however, calculated the transit time dispersion from the photocathode to the deflection plates, assuming the angular distribution is Lambertian. The calculated dispersion is $250 \mathrm{fs}$.

The spatial resolution of the streak tube was simulated by tracing the trajectories of the electrons from the photocathode to the phosphor screen. This was done by solving the dynamic equations of motion for the electrons in an electromagnetic field, using the Runge-Kutta method. The magnetic field distribution was calculated by the finite difference method, assuming that the shield metal has an infinitely high magnetic permeability, and is not saturated. The calculated image width of the slit on the phosphor screen is $\approx 60 \mu \mathrm{m}$.

In our camera, the photoelectrons are multiplied by a microchannel plate (MCP) detector placed in front of the output phosphor screen, as shown in Fig. 1. The output phosphor screen is then fiberoptically coupled to a proximity focused second generation image intensifier. The image is then lens coupled to a low light change coupled device (CCD) camera, which is connected to a frame grabber. The experimentally measured width of the slit image on the CCD camera is $\approx 80 \mu \mathrm{m}$. The high voltage on the streak camera photocathode was applied by superimposing a $-5 \mathrm{kV}$ pulse to a $-5 \mathrm{kV}$ dc voltage. Use of a pulsed extraction field prevents electrical breakdown between the photocathode and the anode. A GaAs photoconductive switch ${ }^{12,13}$ was used to obtain a fast ramp voltage for driving the deflection plates. This switch also exhibits a small relative time jitter of about 5 ps. The resulting sweep speed is $2 \times 10^{8} \mathrm{~m} / \mathrm{s}$, so that the camera time resolution is limited at $400 \mathrm{fs}$ by the spatial resolution and sweep speed. Also taking into account the time dispersion, the total estimated time resolution is $\approx 0.5 \mathrm{ps}$.

Preliminary tests of our camera were performed using the third harmonic of a Ti:sapphire laser. ${ }^{14}$ The $265 \mathrm{~nm}$ light was generated by focusing an $800 \mathrm{~nm}, 26 \mathrm{fs}, 3 \mathrm{~mJ}$ laser pulse in air. They demonstrate a time resolution of $800 \mathrm{fs}$ at this wavelength, as shown in Fig. 2. Two uv pulses were used for calibration purposes. From our uv tests, we found that the sweep speed of the camera could not be increased beyond a certain point, because the electron path was then obstructed by the plates. This occurred because the speed of the electron bunch through the deflection region does not perfectly match the speed of the voltage pulse in the deflection plates in the propagation direction.

To test the time resolution of the camera in the x-ray and xuv region, we used the fastest $\mathrm{x}$-ray pulses available to date, which are high harmonics generated by a 25 fs Ti:sapphire

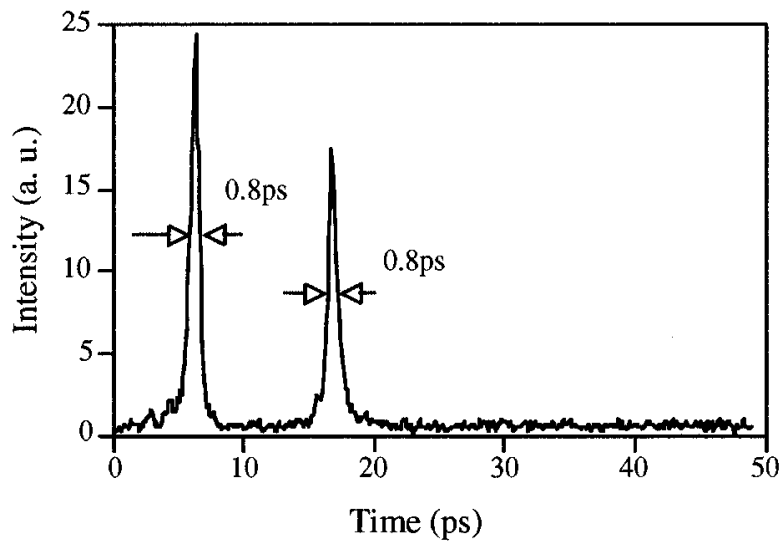

FIG. 2. Streak camera time resolution measured using $15 \mathrm{fs}$ uv pulses at 265 $\mathrm{nm}$.

laser. $^{4}$ A 2-20 mJ laser pulse was focused into a gas cell filled with Ar gas. The generated high harmonics were monochromatized using an $\mathrm{x}$-ray spectrometer. We used a single high harmonic signal centered at $\approx 170 \AA$ for the streak camera calibration. The pulse duration of the high harmonics is believed to be significantly shorter than the 26 fs laser pulse itself. However, dispersion introduced by the $\mathrm{X}$-ray spectrometer broadens the X-ray pulse to $300 \mathrm{fs}$. Using these high harmonics, we obtained a time resolution of 880 fs in the x-ray region for our streak camera, as shown in Fig. 3. This is, to our knowledge, the first demonstration of a sub-ps response x-ray streak camera. Our results are somewhat longer than the expected $0.6 \mathrm{ps}$ response calculated from a convolution of the dispersed $\mathrm{x}$-ray pulse duration $(\approx 0.3 \mathrm{ps})$ and estimated camera resolution $(\approx 0.5 \mathrm{ps})$. We believe that the difference is due to the decrease of spatial resolution in the dynamic or streaked mode. Further work is in progress to improve our results, by designing better electrically matched streak plates.

In conclusion, we have demonstrated a novel design $\mathrm{x}$-ray streak camera with sub-picosecond time resolution. Sub-20 fs high harmonics were used to calibrate our streak camera. We believe that the time resolution our camera can

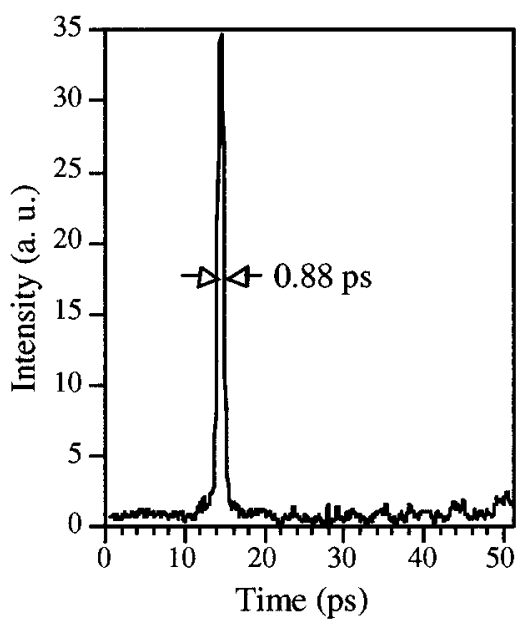

FIG. 3. Streak camera time resolution measured using 300 fs soft-X-ray pulses at $17 \mathrm{~nm}$. 
be improved further by redesigning the deflection plates to achieve higher sweep speeds. Our work demonstrates that sub-picosecond time resolution experiments can now be performed using currently available synchrotron or laserplasma sources.

This work was supported by the National Science Foundation, by the Air Force Office of Scientific Research, and by the Chinese Science and Technology Committee. The authors gratefully acknowledge the assistance of Hou Xun. Zenghu Chang acknowledges fellowship support from the Chinese Academy of Sciences. Henry Kapteyn acknowledges support from a Sloan Foundation Fellowship.

${ }^{1}$ M. M. Murnane, H. C. Kapteyn, M. D. Rosen, and R. W. Falcone, Science 251, 531 (1991).

${ }^{2}$ A. L'Huillier and P. Balcou, Phys. Rev. Lett. 70, 774 (1993).

${ }^{3}$ J. J. Macklin, J. D. Kmetec, and C. L. Gordon III, Phys. Rev. Lett. 70, 766 (1993).
${ }^{4}$ J. Zhou, J. Peatross, M. M. Murnane, H. C. Kapteyn, and I. P. Christov, Phys. Rev. Lett. 76, 752 (1996).

${ }^{5}$ M. M. Murnane, H. C. Kapteyn, and R. W. Falcone, Appl. Phys. Lett. 56, 1948 (1990).

${ }^{6}$ R. Shepherd, R. Booth, D. Price, M. Bowers, D. Swan, J. Bonlie, B. Young, J. Dunn, B. White, and R. Stewart, Rev. Sci. Instrum. 66, 719 (1995).

${ }^{7}$ J. M. Schins, P. Breger, P. Agostini, R. C. Constantinescu, H. G. Muller, G. Grillon, A. Antonetti, and A. Mysyrowicz, Phys. Rev. Lett. 73, 2180 (1994).

${ }^{8}$ J. P. Zhou, C. P. Huang, M. M. Murnane, and H. C. Kapteyn, Opt. Lett. 20, 64 (1995).

${ }^{9}$ G. L. Stradling, J. K. Studebaker, C. Cavailler, J. Launspach, and J. Planes, Proc. SPIE 693, 83 (1986).

${ }^{10}$ D. J. Bradley, A. G. Roddie, W. Sibbett, M. H. Key, M. J. Lamb, C. L. S. Lewis, and P. Sachsenmaier, Opt. Commun. 15, 231 (1975).

${ }^{11}$ B. L. Henke, J. Liesegang, and S. D. Smith, Phys. Rev. B 19, 3004 (1979).

${ }^{12}$ G. A. Mourou and W. Knox, Appl. Phys. Lett. 36, 623 (1980)..

${ }^{13}$ A. Maksimchuk, M. Kim, J. Workman, G. Korn, J. Squire, D. Du, D. Umstadter, and G. Mourou, Rev. Sci. Instrum. 67, 1 (1996).

${ }^{14}$ S. Backus, J. Peatross, M. Murnane, and H. Kapteyn, Opt. Lett. 21, 665 (1996). 